Kiyoshi Shiga

Nagoya Math. J.

Vol. 42 (1971), 57-66

\title{
ON HOLOMORPHIG EXTENSION FROM THE BOUNDARY
}

\author{
KIYOSHI SHIGA
}

\section{Introduction}

Let $D$ be a bounded domain of the complex $n$-space $C^{n}(n \geq 2)$, or more generally a pair $(M, D)$ a finite manifold (c.f. Definition 2.1), and we assume the boundary $\partial D$ is a smooth and connected submanifold. It is well known by Hartogs-Osgood's theorem that every holomorphic function on a neighbourhood of $\partial D$ can be continued holomorphically to $D$. Generalizing the above theorem we shall prove that if a differentiable function on $\partial D$ satisfies certain conditions which are satisfied for the trace of a holomorphic function on a neighbourhood of $\partial D$, then it can be continued holomorphically to $D$ (Theorem 2-5). The above conditions will be called the tangential Cauchy Riemann equations.

Using the above result, we shall determine the condition for a diffeomorphism of $\partial D$ to be continued to a holomorphic automorphism of $D$ (Theorem 3-3). Finally as its corollary the analogy to functions holds for crosssections of a holomorphic vector bundle. (Theorem 3-5)

In preparing this paper, I have received many advices from Professor M. Ise and Professor T. Nagano. I would like to express my cordial thanks to them.

\section{Tangential Cauchy-Riemann equations}

Let $N$ be an $n$-dimensional complex manifold. From now on we always assume $n \geq 2$. Let $M$ be a real smooth submanifold of $N$. We denote by $T_{p}(M)$ the real tangent space of $M$ at $p$. Let $J$ be the complex structure of $N$.

$$
C_{p}=T_{p}(M) \cap J T_{p}(M)
$$

is the maximum complex subspace of $T_{p}(M)$, and we denote its complex dimension by $m(p)$ and we assume $m(p)$ is constant on $M$.

Received March 13, 1970. 
Then $T_{p}(M) \otimes C$ is decomposed to

$$
T_{p}(M) \otimes C=H_{p}+\bar{H}_{p}+L_{p} \text { (direct sum) }
$$

where

$$
\begin{aligned}
& H_{p}=\left\{X \in T_{p}(M) \otimes C ; X \text { is a } \sqrt{-1} \text { eigen vector of } J\right\} \\
& \bar{H}_{p}=\left\{X \in T_{p}(M) \otimes C ; X \text { is a }-\sqrt{-1} \text { eigen vector of } J\right\},
\end{aligned}
$$

and $L_{p}$ is a complemental subspace of $H_{p}+\bar{H}_{p}$. We call an element of $H_{p}$, $\bar{H}_{p}$, holomorphic and anti-holomorphic tangent vector respectively. It is evident that $\left(\overline{H_{p}}\right)=\bar{H}_{p}$, where the upper bar means complex conjugate with respect to $T_{p}(M)$, and that $\operatorname{dim}_{\boldsymbol{C}} H_{p}=\operatorname{dim}_{\boldsymbol{C}} \bar{H}_{p}=m(p)$. Now we define

Definition 1-1. Let $f$ be a complex valued differentiable function defined on a neighbourhood of $p \in M$. If $X f=0$ for every $X \in \bar{H}_{p}$, we call that $f$ satisfies the tangential Cauchy-Riemann equations at $p$.

If $f$ satisfies the tangential Cauchy-Riemann equations at every point of the domain of $f$, we call $f$ satisfies the tangential Cauchy-Riemann equations (in short, $T-C-R$ equations).

In the following we consider only the case when $M$ is a real hypersurface of $N$. In this case we define

Definition 1-2. Let $M$ be a real hypersurface of $N$. We call a real valued differentiable function $\varphi$ a defining function of $M$ if it satisfies the following conditions.

1). $M=\{z \in N ; \varphi(z)=0\}$

2). $\operatorname{grad} \varphi$ does not vanish on $M$.

Let $\varphi$ be a defining function of $M$ and $p_{0}$ a point of $M$. Let $\left(z_{1}, \cdots, z_{n}\right)$ be a local coordinate system at $p_{0}$. Since $\operatorname{grad} \varphi$ does not vanish on $M$, then we can assume $\varphi_{\bar{z}_{n}}:=\frac{\partial \varphi}{\partial \bar{z}_{n}}$ does not vanish on some neighbourhood $U$ of $p_{0}$. We can choose a base of $H_{p}, \bar{H}_{p}$, and $L_{p}$ at $p \in U$ as following

$$
H_{p}:\left\{\begin{array}{l}
\left(X_{1}\right)_{p}=\left(\varphi_{z_{n}}\right)_{p}\left(\frac{\partial}{\partial z_{1}}\right)_{p}-\left(\varphi_{z_{1}}\right)_{p}\left(\frac{\partial}{\partial z_{n}}\right)_{p} \\
\cdot \cdot \cdot \cdot \\
\left(X_{n-1}\right)_{p}=\left(\varphi_{z_{n}}\right)_{p}\left(\frac{\partial}{\partial z_{n-1}}\right)_{p}-\left(\varphi_{z_{n-1}}\right)_{p}\left(\frac{\partial}{\partial z_{n}}\right)_{p}
\end{array}\right.
$$




$$
\begin{gathered}
\bar{H}_{p}:\left\{\begin{array}{l}
\left(\bar{X}_{1}\right)_{p}=\left(\varphi_{\bar{z}_{n}}\right)_{p}\left(\frac{\partial}{\partial \bar{z}_{1}}\right)_{p}-\left(\varphi_{\bar{z}_{1}}\right)_{p}\left(\frac{\partial}{\partial \bar{z}_{n}}\right)_{p} \\
\cdot \cdot \cdot \cdot \\
\left(\bar{X}_{n-1}\right)_{p}=\left(\varphi_{\bar{z}_{n}}\right)_{p}\left(\frac{\partial}{\partial \bar{z}_{n-1}}\right)_{p}-\left(\varphi_{\bar{z}_{n-1}}\right)_{p}\left(\frac{\partial}{\partial \bar{z}_{n}}\right)_{p}
\end{array}\right. \\
L_{p}: \quad Y_{p}=\left(\varphi_{\bar{z}_{n}}\right)_{p}\left(\frac{\partial}{\partial z_{n}}\right)_{p}-\left(\varphi_{z_{n}}\right)_{p}\left(\frac{\partial}{\partial \bar{z}_{n}}\right)_{p}
\end{gathered}
$$

It means $H=\bigcup_{p \in M} H_{p}, \bar{H}=\bigcup_{p \in M} \bar{H}_{p}$ are subbundles of $T(M) \otimes C$.

\section{Holomorphic extension of functions.}

Let $M$ be a Stein manifold and $D$ be a domain of $M$. Now we introduce the following definition.

Definition 2-1. A pair $(M, D)$ is called a finite manifold, if the following conditions are satisfied.

0). $\quad M$ is a Stein manifold and $\operatorname{dim} M \geq 2$

1). $D$ is a connected relatively compact domain of $M$.

2). the boundary of $D$, denoted by $\partial D$, is a connected smooth real hypersurface of $M$.

Let $(M, D)$ be a finite manifold. We use the following notations.

$$
\begin{aligned}
& C^{\infty}(\bar{D})=\{\text { a differentiable function on } \bar{D}\} \\
& H(\bar{D})=\left\{f \in C^{\infty}(\bar{D}) ;\left.f\right|_{D} \text { is a holomorphic function }\right\}
\end{aligned}
$$

where $\left.f\right|_{D}$ is the restriction of $f$ to $D$.

We choose a defining function $\varphi$ of $\partial D$ such that

$$
D=\{z ; \varphi(z)<0\} \text { and } M-D=\{z ; \varphi(z) \geq 0\}
$$

Since $\varphi$ is a defining function, grad $\varphi$ does not vanish on $\partial D$.

It is convenient to express the $T-C-R$ equations in another way. Let $f$ be a differentiable function on $\partial D$. There exists $F \in C^{\infty}(\bar{D})$ so that $F \mid \partial D=f$.

Lemma 2-2. A differentiable function $f$ on $\partial D$ satisfies $T-C-R$ equations if and only if $\bar{\partial} F \wedge \bar{\partial} \varphi=0$ on $\partial D$, where $F$ is a differentiable function on $\bar{D}$ as above and $\bar{\partial}$ is the Cauchy-Riemann operator.

Proof is clear from Definition 1-1. 
Lemma 2-3. (Hörmander [1] p. 137) Let $M$ be a Stein manifold and $\alpha$ $a(0,1)$ type 1 -form of class $C^{k}$. If $\bar{\partial} \alpha=0$, there exists $a k-n$ time differentiable function $u$ such that $\bar{\partial} u=\alpha$.

We shall prove the following corollary, using the above lemma.

Corollary 2-4. Let $\alpha$ be a $(0,1)$ type 1-form of class $C^{k}$ on a Stein manifold $M$. If $\bar{\partial} \alpha=0$ and $K=\operatorname{supp} \alpha$ is compact and $M-K$ is connected, there exists $k-n$ time differentiable function $u$ so that $\bar{\partial} u=\alpha$ and supp $u \subset K$.

Proof. There exists a $k-n$ time differentiable function $v$ such that $\bar{\partial} v=\alpha$ by lemma 2-3. Since $\bar{\partial} v=0$ on $M-K, v$ is holomorphic on $M-K$. By Hartogs-Osgood's theorem (Kasahara [2]) $\left.v\right|_{M-K}$ can be continued to a holomorphic function $w$ on $M$. We put $u=v-w$, it follows that $\bar{\partial} u=\bar{\partial} v-\bar{\partial} w$ $=\bar{\partial} v=\alpha$, and supp $u \subset K$.

We shall prove the following theorem by the method of Hörmander [1].

Theorem 2-5. Let $(M, D)$ be a finite manifold, and $f$ a differentiable function on $\partial D$. If $f$ satisfies $T-C-R$ equations, there exists $\tilde{f} \in H(\bar{D})$ such that $\tilde{f} \mid \partial D=f$.

Proof. (1-st step) We construct by induction a differentiable function $U_{k} \in C^{\infty}(\vec{D})$ for every positive integer $k$ which satisfies the following conditions;

$$
\left.U_{k}\right|_{\partial D}=f \text { and } \bar{\partial} U_{k}=0\left(\varphi^{k}\right) \text {. }
$$

We extend $f$ to a function on $\bar{D}$ as an element of $C^{\infty}(\bar{D})$, and we denote it by $f$ also. By lemma $2-2 \bar{\partial} f \wedge \bar{\partial} \varphi=0$ on $\partial D$. Then we can decompose $\bar{\partial} f$ as

$$
\bar{\partial} f=h_{1} \bar{\partial} \varphi+\varphi h_{2}
$$

where $h_{1} \in C^{\infty}(\bar{D})$ and $h_{2}$ is a differentiable $(0,1)$ type 1 -form. We write it by $h_{2} \in C_{(0,1)}^{\infty}(\bar{D})$ in the following.

By simple calculation we have

$$
\begin{aligned}
\bar{\partial}\left(f-h_{1} \varphi\right) & =\bar{\partial} f-\left(\bar{\partial} h_{1}\right) \varphi-h_{1} \bar{\partial} \varphi \\
& =\varphi h_{2}-\left(\bar{\partial} h_{1}\right) \varphi \\
& =\varphi\left(h_{2}-\bar{\partial} h_{1}\right) .
\end{aligned}
$$

Put $U_{1}:=f-\varphi h_{1}$, then $\left.U_{1}\right|_{\partial D}=f$ and $\bar{\partial} U_{1}=0(\varphi)$. We have thus constructed $U_{1}$. 
Now we assume that $U_{k-1}$ is constructed, i.e.

$$
\left.U_{k-1}\right|_{\partial D}=f, \quad \bar{\partial} U_{k-1}=0\left(\varphi^{k-1}\right) .
$$

Then we can write $\bar{\partial} U_{k-1}=\varphi^{k-1} h, h \in C_{(0,1)}^{\infty}(\bar{D})$. Then

$$
\begin{aligned}
\bar{\partial} \bar{\partial} U_{k-1} & =0 \\
& =(k-1) \varphi^{k-2} \bar{\partial} \varphi \wedge h+\varphi^{k-1} \bar{\partial} h \\
& =\varphi^{k-2}((k-1) \bar{\partial} \varphi \wedge h+\varphi \cdot \bar{\partial} h)
\end{aligned}
$$

Hence $(k-1) \bar{\partial} \varphi \wedge h+\varphi \bar{\partial} h=0$. However $\varphi \bar{\partial} h$ vanishes on $\partial D$, so that $h$ must satisfies $\bar{\partial} \varphi \wedge h=0$ on $\partial D$.

This imples that $h=\bar{\partial} \varphi \wedge h_{2 k-1}+\varphi h_{2 k}$, where $h_{2 k-1} \in C^{\infty}(\bar{D}), \quad h_{2 k} \in C_{(0,1)}^{\infty}(\bar{D})$. Put $U_{k}:=U_{k-1}-\left(\frac{1}{k} \cdot \varphi^{k}\right) h_{2 k-1}$. We see that the function $U_{k}$ satisfies the condition $(2-1)$, because

$$
\begin{aligned}
\bar{\partial} U_{k} & =\bar{\partial} U_{k-1}-\left(\varphi^{k-1} \bar{\partial} \varphi\right) h_{2 k-1}-\left(\frac{1}{k} \cdot \varphi^{k}\right) \bar{\partial} h_{2 k-1} \\
& =\varphi^{k} \cdot h_{2 k}-\left(\frac{1}{k} \cdot \varphi^{k}\right) \bar{\partial} h_{2 k-1} \\
& =\varphi^{k}\left(h_{2 k}-\frac{1}{k} \bar{\partial} h_{2 k-1}\right) .
\end{aligned}
$$

(2-nd step) Let $k \geq n+2$. We define $v_{k} \in C_{(0,0)}^{k}(M)$ with

$$
\left.v_{k}\right|_{\bar{D}}=\bar{\partial} U_{k} \text { and }\left.\quad v_{k}\right|_{M-\bar{D}}=0 .
$$

Note that supp. $\quad v_{k} \subset \bar{D}$. By corollary 2-4 there exists $w_{k} \in C^{k-1-n}(M)$ which satisfies $\bar{\partial} w_{k}=v_{k}$ and supp. $w_{k} \subset \bar{D}$. Put $f_{k}=U_{k}-w_{k}$. Then we have $f_{k} \in C^{(k-1-n)}(\bar{D}),\left.f_{k}\right|_{\partial D}=f$ and $\bar{\partial} f_{k}=\bar{\partial} U_{k}-\bar{\partial} U_{k}-\bar{\partial} w_{k}=0$. Thus $f_{k}$ is holomorphic on $D$ and its boundary value is $f$. Then by the uniqueness of continuation

$$
f_{k}=f_{k+1}=f_{k+2}=\cdots
$$

We put $\tilde{f}=f_{k}=f_{k+1}=f_{k+2}=\cdots$, it is the desired one.

Q.E.D.

\section{Holomorphic extension of mappings}

Let $M$ be a complex manifold and $S$ a real hypersurface of $M$. As we saw in $\S 1, T_{p}(S) \otimes C$ is decomposed at $p \in S$ as follows:

$$
T_{p}(S) \otimes C=H_{p}+\bar{H}_{p}+L_{p} \quad \text { (direct sum) }
$$

where $H_{p}, \bar{H}_{p}$, are holomorphic and anti-holomorphic tangent space at $p$, 
respectively. Here we define the tangential Cauchy-Riemann equations for mapping.

Definition $3-1$. Let $M, M^{\prime}$, be complex manifolds and $S, S^{\prime}$ real hypersurfaces of $M, M^{\prime}$, respectively. Let $\mu$ be a differentiable mapping from $S$ to $S^{\prime}$. The following conditions 1$\left.\left.), 1^{\prime}\right), 2\right), 3$ ) are equivalent. If $\mu$ satisfies one of the conditions, we say that $\mu$ satisfies the tangential CauchyRiemann equations (in short, $T-C-R$ equations).

1). $\mu_{*}\left(H_{p}(S)\right) \subset H_{\mu(p)}\left(S^{\prime}\right)$ for every point $p \in S$

1)'. $\quad \mu_{*}\left(\bar{H}_{p}(S)\right) \subset \bar{H}_{\mu(p)}\left(S^{\prime}\right)$ for every point $p \in S$

2). a differentiable function $f$ on an open set of $S^{\prime}$ satisfies $T-C-R$ equations, then $\mu^{*} f$ satisfies $T-C-R$ equations on its domain.

3). Let $\left(z_{1}^{\prime}, \cdots, z_{m}^{\prime}\right)$ be a local coordinate system at $q=\mu(p)$ of $M$. Then $f_{i}:=\mu^{*} z_{i}^{\prime}:(i=1, \cdots, m)$ satisfies $T-C-R$ equations.

We shall prove that four conditions of definition are equivalent.

$\left.1) \Longrightarrow 1^{\prime}\right)$. We choose a local coordinate system $\left(z_{1}, \cdots, z_{n}\right)$ of $M$ at $p$ as follows.

$$
H_{p}=\left\{\left\{\left(\frac{\partial}{\partial z_{1}}\right)_{p}, \cdots,\left(\frac{\partial}{\partial z_{n-1}}\right)\right\}\right\}, \bar{H}_{p}=\left\{\left\{\left(\frac{\partial}{\partial \bar{z}_{1}}\right)_{p}, \cdots,\left(\frac{\partial}{\partial \bar{z}_{n-1}}\right)_{p}\right\}\right\}
$$

Take some local coordinate system $\left(z_{1}^{\prime}, \cdots, z_{m}^{\prime}\right)$ of $M^{\prime}$ at $q=\mu(p)$ and put $f_{i}=\mu^{*} z_{i}^{\prime}$, then

$$
\mu_{*}\left(\frac{\partial}{\partial z_{i}}\right)_{p}=\sum_{j}\left(\frac{\partial f_{j}}{\partial z_{i}}\right)_{p}\left(\frac{\partial}{\partial z_{j}^{\prime}}\right)_{\mu(p)}+\sum_{j}\left(\frac{\partial \bar{f}_{j}}{\partial z_{i}}\right)_{p}\left(\frac{\partial}{\partial \bar{z}_{j}^{\prime}}\right)_{\mu(p)} \quad i=1, \cdots, n
$$

But from the condition 1) $\mu_{*}\left(\frac{\partial}{\partial z_{i}}\right) \in H\left(S^{\prime}\right)$, so that

$$
\left(\frac{\partial \bar{f}_{j}}{\partial z_{i}}\right)_{p}=\left(\frac{\overline{\partial f_{j}}}{\partial \bar{z}_{i}}\right)_{p}=0 \quad j=1, \cdots, m
$$

Hence it follows that

$$
\begin{aligned}
\mu_{*}\left(\frac{\partial}{\partial \bar{z}_{i}}\right)_{p} & =\sum_{j=1}^{m}\left(\frac{\partial f_{j}}{\partial \bar{z}_{i}}\right)_{p}\left(\frac{\partial}{\partial z_{j}^{\prime}}\right)_{\mu(p)}+\sum_{j=1}^{m}\left(\frac{\partial \bar{f}_{j}}{\partial \bar{z}_{i}}\right)_{p}\left(\frac{\partial}{\partial \bar{z}_{j}^{\prime}}\right)_{\mu(p)} \\
& =\sum_{j=1}^{m}\left(\frac{\partial \bar{f}_{j}}{\partial \bar{z}_{i}}\right)_{p}\left(\frac{\partial}{\partial \bar{z}_{j}^{\prime}}\right)_{\mu(p)} \in \bar{H}_{\mu(p)}\left(S^{\prime}\right)
\end{aligned}
$$

$\left.1^{\prime}\right) \Longrightarrow 1$ ) is now obvious. 
2) $\Longrightarrow 3)$. Since $\left(z_{1}^{\prime}, \cdots, z_{m}^{\prime}\right)$ is a local coordinate of $M^{\prime}$ at $\mu(p)=q$, it is trivial that $z_{i}^{\prime}$ satisfies $T-C-R$ equations. By condition 2), $f_{i}=\mu_{*}\left(z_{i}^{\prime}\right)$ satisfies $T-C-R$ equations.

$1) \Longrightarrow 2$ ). Let $g$ be a differentiable function defined on a neighbourhood (in $S^{\prime}$ ) of $q=\mu(p)$ which satisfies $T-C-R$ equations. Let $X$ be any element of $\bar{H}_{p}(S)$. By $\left.1^{\prime}\right) \mu_{*} X \in \bar{H}_{\mu(p)}\left(S^{\prime}\right)$, and $X\left(\mu^{*} g\right)=\left(\mu_{*} X\right) g=0$. Thus $g$ satisfies $T-C-R$ equations.

$3) \Longrightarrow 1$ ). We choose a local coordinate system at $p$ as above. We also have

$$
\mu_{*}\left(\frac{\partial}{\partial z_{i}}\right)_{p}=\sum_{j=1}^{m}\left(\frac{\partial f_{j}}{\partial z_{i}}\right)_{p}\left(\frac{\partial}{\partial z_{i}^{\prime}}\right)_{\mu(p)}+\left(\frac{\partial \bar{f}_{j}}{\partial z_{i}}\right)_{p}\left(\frac{\partial}{\partial \bar{z}_{j}}\right)_{\mu(p)} \quad(1 \leq i \leq n-1)
$$

Since $f$ satisfies $T-C-R$ equations, we have $\left(\frac{\partial f_{j}}{\partial \bar{z}_{i}}\right)_{p}=\overline{\left(\frac{\partial \bar{f}_{j}}{\partial z_{i}}\right)}=0$. Then $\mu_{*}\left(\frac{\partial}{\partial z_{i}}\right)_{p} \in H_{\mu(p)}\left(S^{\prime}\right)$. This means $\mu_{*}\left(H_{p}(S)\right) \subset H_{\mu(p)}\left(S^{\prime}\right)$.

Lemma 3-2. Let $M$ be a complex manifold and $S$ be a real hypersurface of $M$. The set of all diffeomorphisms of $S$ which satisfies $T-C-R$ equations is a group.

Proof is clear by the condition 1) of Definition 3-1. But I don't know the group of lemma $3-2$ is a Lie group or not.

Let $(M, D)$ be a finite manifold. We introduce the following notations.

Let $\operatorname{Diff}(\bar{D})$ be the group of all $C^{\infty}$-diffeomorphisms of $\bar{D}$, and

Aut $(\bar{D})=\left\{\mu \in \operatorname{Diff}(\bar{D}) ;\left.\mu\right|_{D}\right.$ is a holomorphic automorphism of $\left.D\right\}$

Now we shall prove the following

Theorem 3-3. If a diffeomorphism $\mu: \hat{o} D \rightarrow \partial D$ satisfies $T-C-R$ equations, there exists $\tilde{\mu} \in \operatorname{Aut}(\bar{D})$ such that $\left.\tilde{\mu}\right|_{\partial D}=\mu$.

Proof. Let $p$ be any point of $\partial D$. Since $M$ is a Stein manifold, there is a local coordinate system $\left(f_{1}, \cdots, f_{n}\right)$ of $M$ at $q=\mu(p)$, where $f_{1}, \cdots, f_{n}$ are holomorphic functions on $M$. By definition 3-1 $\mu^{*} f_{i}$ satisfies $T-C-R$ equations. Then by theorem $2-5$ there exist $\tilde{f}_{i} \in H(\bar{D})$ such that $\left.\tilde{f}_{i}\right|_{\partial D}=\mu^{*} f_{i}$. We take a sufficiently small neighbourhood $U_{p}$ of $p$, and define the mapping $\mu_{U p}: U_{p} \cap \bar{D} \rightarrow M$, using the above local coordinate system $\left(f_{1}, \cdots, f_{n}\right)$ at $q$, by

$$
\mu_{U_{p}}\left(p^{\prime}\right)=\left(\tilde{f}_{1}\left(p^{\prime}\right), \cdots, \tilde{f}_{n}(p)\right), \quad p^{\prime} \in U_{p} \cap \bar{D}
$$

By the uniqueness of the holomorphic continuation of functions, there exist 
a small neighbourhood $U$ of $\partial D$, so that $U \cap \vec{D}$ is connected, and there exists a holomorphic mapping

$$
\mu_{U}: \bar{D} \cap U \rightarrow M \text { with }\left.\mu_{U}\right|_{U_{p}} \cap_{\bar{D}}=\mu_{U_{p}}
$$

Since $D-D \cap U$ is compact, there exists a holomorphic mapping $\mu: D \rightarrow M$ so that $\left.\tilde{\mu}\right|_{D \cap U}=\tilde{\mu}_{U}$ by Hartogs-Osgood's theorem (K. Kasahara [2]). We shall prove that the mapping $\tilde{\mu}$ is the desired one.

By the construction of $\tilde{\mu}, \tilde{\mu}$ is holomorphic on $D$ and $\left.\tilde{\mu}\right|_{\partial D}=\mu$. First we shall prove the rank of $\tilde{\mu}$ is $2 n$ at each point of a neighbourhood of $\partial D$ in $\bar{D}$. Here we may assume that there exist real vector fields $X_{1}, \cdots, X_{n}$, $J X_{1}, \cdots, J X_{n-1}$ on a small neighbourhood $V_{p_{0}}$ of $p_{0}$ in $\partial D$, such that they form a base of $T_{p}(\partial D)$ at every point $p$ of $V_{p_{0}}$. We can construct them taking real parts of the base of $H$ and a real vector contained in $L$ given in $\$ 1$.

We extend $X_{1}, \cdots, X_{n}$ to a neighbourhood $W_{p_{0}}$ of $V_{p_{0}}$ and we denote them $\tilde{X}_{1}, \cdots, \tilde{X}_{n}$ and we can assume $\tilde{X}_{1}, \cdots, \tilde{X}_{n}, J \tilde{X}_{1}, \cdots, J \tilde{X}_{n-1}$ are linearly independent at each point of $W_{p_{0}}$, taking $W_{p_{0}}$ sufficiently small. Since $\mu$ is a diffeomorphisms, $\mu_{*}\left(X_{1}\right), \mu_{*}\left(X_{2}\right), \cdots, \mu_{*}\left(X_{n}\right), \mu_{*}\left(J X_{1}\right), \cdots, \mu_{*}\left(J X_{n-1}\right)$ are linearly independent at each point of $\mu\left(V_{p}\right)$, and hence $\tilde{\mu}_{*}\left(\tilde{X}_{1}\right), \cdots, \tilde{\mu}_{*}\left(\tilde{X}_{n}\right)$, $\tilde{\mu}_{*}\left(J \tilde{X}_{1}\right), \cdots, \tilde{\mu}_{*}\left(J \tilde{X}_{n-1}\right)$ are independent at every point of $\tilde{\mu}\left(W_{p} \cap D\right)$, changing $W_{p_{0}}$ smaller if necessary. Since $\tilde{\mu}$ is holomorphic on $D$,

$$
\tilde{\mu}_{*}\left(J \tilde{X}_{i}\right)=J \tilde{\mu}_{*}\left(\tilde{X}_{i}\right), \quad 1 \leq i \leq n
$$

Then $\tilde{\mu}_{*}\left(\tilde{X}_{1}\right), \tilde{\mu}_{*}\left(\tilde{X}_{2}\right), \cdots, \tilde{\mu}_{*}\left(\tilde{X}_{n}\right), \tilde{\mu}_{*}\left(J \tilde{X}_{1}\right), \cdots, \tilde{\mu}_{*}\left(J \tilde{X}_{n}\right)$ are independent at $\tilde{\mu}\left(W_{p} \cap D\right)$. It means the rank of $\tilde{\mu}$ is $2 n$ on $W_{p_{0}} \cap D$. Since $p_{0}$ is an arbitrary point of $\partial D$, there exists a neighbourhood $W$ of $\partial D$ such taht rank of $\tilde{\mu}$ is $2 n$ on $W \cap D$. Hence the set of all ponits of $D$ where rank of $\tilde{\mu}$ is smaller than $2 n$ is a compact analytic set of dimension $n-1 \geq 1$ of $M$. Since $M$ is a Stein manifold, there is no compact analytic set of dimension $n-1 \geq 1$ of $M$. Then $\operatorname{rank} \tilde{\mu}$ is $2 n$ at each point of $D$. Hence $\tilde{\mu}$ is a local diffeomorphism on $D$.

Next we see that $\tilde{\mu}(\bar{D}) \subset \bar{D}$. In fact, if $\tilde{\mu}(\bar{D}) \not \subset \bar{D}$, there is a boundary point $q$ of $\tilde{\mu}(\vec{D})$ such that $q=\tilde{\mu}(p) \notin \widetilde{D}$. Since $\tilde{\mu}(\partial D)=\partial D$, we have $p \in D$. This contradicts to the fact $\tilde{\mu}$ is a local diffeomorphism at $p$.

Since $\mu^{-1}$ also satisfies $T-C-R$ equations by Lemma $3-2$, theer is $\left(\widetilde{\mu^{-1}}\right)$ such that $\left.\left(\widetilde{\mu^{-1}}\right)\right|_{D}$ is holomorphic and $\left.\left(\widetilde{\mu^{-1}}\right)\right|_{\partial D}=\mu^{-1}$. Since $\tilde{\mu}(\bar{D}) \subset \bar{D}$ and 
$\left(\widetilde{\mu^{-1}}\right)(\bar{D}) \subset \bar{D}$, we have $(\tilde{\mu})\left(\widetilde{\mu^{-1}}\right)=i d=\widetilde{i d}$, and $\left(\widetilde{\mu^{-1}}\right)(\tilde{\mu})=i d=\widetilde{i d}$. This means that $\tilde{\mu}$ is a holomorphic automorphism of $D$.

By the proof of the above theorem, we conclude the following theorem.

Theorem 3-4. Let $(M, D)$ be a finite manifold, $N$ a Stein manifold and $S$ a real hypersurface of $N$. If a mapping $\mu: \partial D \rightarrow S$ satisfies $T-C-R$ equations, there exists a differentiable mapping $\tilde{\mu}: \bar{D} \rightarrow N$ such that $\left.\tilde{\mu}\right|_{\partial D}=\mu$ and $\left.\tilde{\mu}\right|_{D}$ is holomorphic.

In the above theorem the conditoin that $S$ is a real hypersurface can be changed to that $\mu: \partial D \rightarrow N$ satisfies the condition 1) of Definition 3-1.

By using the above theorem, we consider the holomorphic extension of a differentiable cross-section of a holomorphic fibre bundle.

Let $(M, D)$ be a finite manifold and $E$ a holomorphic fibre bundle over $M$. If a differentiable cross-section $s$ over $\partial D$ satisfies $T-C-R$ equations as a mapping $s: \partial D \rightarrow E$, we call $s$ satisfies the tangential Cauchy-Reimann equations, (in short, $T-C-R$ equations).

THeORem 3-5. If a differentiable cross-section $s$ over $\partial D$ of a holomorphic fibre bundle whose fibre is a Stein manifold, satisfies $T-C-R$ equations, there exists a differentiable cross-section $\tilde{s}$ over $\bar{D}$ such that $\left.\tilde{s}\right|_{\partial D}=s$ and $\left.\tilde{s}\right|_{D}$ is a holomorphic crosssection.

Proof. Since $M$ and the fibre of $E$ are Stein manifolds, $E$ is also a Stein manifold by the theorem of Matsushima-Morimoto [3]. Since cross-section $s$ satisfies $T-C-R$ equations, there exists a mapping $\tilde{s}: D \rightarrow E$ such that $\left.\tilde{s}\right|_{\partial D}=s$ and $\left.\tilde{s}\right|_{D}$ is holomprohic by Theorem 3-4.

Then it sufficies to prove $\tilde{s}$ is a cross-section i.e. $\pi \tilde{s}=i d$ where $\pi$ is the projection from $E$ to $M$.

$\tilde{f}=(\pi \tilde{s})^{*} f$ is a holomorphic function for every $f \in H(\bar{D})$. It is clear that $\left.\tilde{f}\right|_{\partial D}=f$ implies $\tilde{f}=(\pi \tilde{s})^{*} f=f$ on $D$. By considering coordinate functions, it means $\pi \tilde{s}=i d$.

Remark 3.6. If $E$ is a holomorphic vector bundle, $E$ is a Stien manifold since vector space over $\boldsymbol{C}$ is a Stein manifold. In this case if a differentiable cross-section $s$ over $\partial D$ satisfies $T-C-R$ equations, by the local expression, then it satisfies $T-C-R$ equations as cross-section. Then $s$ can be holomorphically extended to the cross-section over $\tilde{D}$ by the above theorem. 


\section{BiBLIOGRAPHY}

[1] Hörmander, L. An introduction to complex analysis in several variables. Van Norstrand 1966.

[2] Kasahara, K. On Hartogs-Osgood's theorem for Stein Spaces., J. Math. Soci. Japan 17 (1965) pp. 297-314.

[ 3 ] Matsushima, Y. and Morimoto, A. Sur certains espaces fibrés holomorphes sur une variété de Stein., Bull. Soci. Math. France 88, 1960 pp. 137-155.

Nagoya University 